\title{
Computer-Assisted Regulation of Emotional and Social Processes
}

\author{
Toni Vanhala and Veikko Surakka \\ Research Group for Emotions, Sociality, and Computing, TAUCHI, \\ Department of Computer Sciences, University of Tampere \\ Finland
}

\section{Introduction}

Imagine a person who has a fear of other people. Let us call her Anna. She is afraid of people watching her every move as she stands in a line or walks down the street. Meeting new people is almost impossible as she always feels stared at and judged by everyone. This fear, or maybe even a phobia, can make Anna's life very complicated. It is difficult for her to travel through public spaces in order to get to work, to deal with a bus or taxi driver, shop for groceries, etc. Anna's leisure time activities are also very limited. The situation is indeed a vicious cycle, as it is even difficult for her to seek treatment and go to a therapist.

In USA alone, there are approximately 15 million people like Anna who suffer from social anxiety disorder (Anxiety Disorders Association of America, 2008). A total of 40 million people suffer from different anxiety disorders. The associated yearly costs of mental health care exceed 42 billion U.S. dollars. Thus, emotional disorders are a significant public health issue. There is a need for demonstrably effective and efficient new methods for therapy.

Computer systems have recently been applied to the treatment of many emotional disorders, including different phobias (Krijn et al., 2004; Wiederhold \& Bullinger, 2005). These systems provide controlled virtual exposure to the object of the disorder, for example, a computer simulation of a spider or a room filled with other people. In this form of behavioural therapy, patients are systematically desensitized by gradual exposure to a computer generated representation of the object of their fear (Weiten, 2007; Krijn et al., 2004). At first, the level of exposure is kept mild and constant, for example, by keeping the object of the fear visually distant and far away. Then, the level of exposure is increased little by little, for example, by moving a virtual spider closer or increasing the number of virtual people. The underlying theory is that such exposure replaces anxiety provoking memories and thoughts with more neutral ones that are acquired in a safe, controlled environment.

It has been shown that people react to computer generated stimuli in the same manner as to authentic, real-life stimuli. For example, socially anxious people are cautious about disturbing embodied artificial characters in virtual reality (Garau et al., 2005). People have also reported higher anxiety and shown increased somatic responses when speaking to negative as compared to neutral and positive audiences consisting of virtual agent characters (Pertaub et al., 2002). As these studies have shown that virtual characters are able to evoke emotions or anxiety, computer generated stimuli show clear potential as a new method for treating different social and emotional disorders by enabling controlled exposure to anxiety provoking stimuli. 
Advantages of computer generated stimuli include accurate control of the grade of exposure, the relative easiness of creating diverse stimuli and varying their characteristics, and the cost-efficiency of therapy. For example, a person who has a phobia of flying can experience a whole virtual air plane trip from take-off to landing at a relatively low cost. Further, the experience can be replicated again and again with small variations to factors that would be very difficult to control in real situations. Virtual environments even allow the re-enactment of traumatic episodes, such as bombings and car accidents. In fact, there are various conditions that have been successfully treated using virtual exposure to artificial stimuli, including fear of flying, fear of driving, fear of confined spaces, fear of public speaking, social phobia, post-traumatic disorders, and panic disorders (Krijn et al., 2004; Wiederhold \& Bullinger, 2005). However, Krijn et al. (2004) concluded in their review of virtual exposure methods that there is little conclusive evidence about the relative effectiveness of virtual reality and real, in vivo exposure. One particular concern was the lack of evidence for the effectiveness of virtual exposure therapy as a stand-alone treatment.

There is evidence that the effectiveness of exposure therapy can be further improved by applying physiological measurements (Wiederhold \& Wiederhold, 2003). For example, physiological signals can be registered and displayed to the patient during exposure therapy (Wiederhold \& Bullinger, 2005; Wiederhold \& Wiederhold, 2003). This way, the patient can gain awareness of physiological processes and learn to voluntarily control them. Voluntary control of emotion-related physiological functions has been shown to influence emotional reactions associated with, for example, fear and facial expressions (Vanhala \& Surakka, 2007a; Wiederhold \& Wiederhold, 2003). In other types of setups, the clinician can monitor these signals, estimate the progress of therapy, and adjust its intensity accordingly.

Previous research has established a number of physiology-based measures of emotional states that can be used as a basis for adapting the therapy (Vanhala \& Surakka, 2007a; Vanhala \& Surakka, 2007b; Partala et al., 2006; Anttonen \& Surakka, 2005; Wilhelm et al., 2006; Surakka \& Vanhala, accepted). These measures include electrical brain and facial muscle activity, heart rate, respiration, and skin conductivity. However, it is not possible to use a single measure as an index of emotional states, as each individual measure is affected by a number of psychological and physiological factors (Ward \& Marsden, 2003). Emotions themselves are often categorized according to a number of dimensions, such as arousal and emotional valence (Bradley \& Lang, 1994). Further, emotional processes are tightly interconnected with other psychophysiological processes, including cognition and attention (Surakka et al., 1998). Thus, it is necessary to take other psychophysiological processes (e.g., attention) into account when recognizing and analyzing emotions (Ward \& Marsden, 2003).

As multi-signal, online monitoring of human psychophysiology involves signals with several varying characteristics (e.g. sample rate and frequency content) and as each measure reflects several inter-linked physiological systems, the amount of information can easily overwhelm a human operator. One way to deal with this challenge is to build perceptual intelligence into computers themselves (Pentland, 2000). Signal analysis of measured psychophysiological signals and states could be performed automatically. Further, the role of human actors in this kind of a virtual therapy system could be changed. Currently, humans need to process all information that is used to control the parameters of a virtual therapy system. Proactive computing could be used to remove this bottleneck (Tennenhouse, 2000). A system that responds to the emotional and physiological state of a person could automatically adapt the computer system according to the rules of desensitization. This way, both the person being treated and the therapist could focus on training to regulate emotions instead of actively interpreting and estimating them. 
The main goal of the present work is to present a new model for computer systems that proactively support emotion regulation. First, in the next section we present examples of single and compound measures of psychophysiological states that could be used to build perceptual intelligence for this kind of a system. Then, in the following section we discuss studies demonstrating the effectiveness of computers in regulating emotions. In the process we identify several computer-generated stimuli that could be used to influence emotional and social processes. In the fourth section we combine these findings into a model that supports both computer-assisted regulation and voluntary control of emotion related psychological and physiological processes. Finally, we discuss the advantages and challenges of this model and suggest pertinent research areas for future work.

\section{Measuring emotions}

As our aim is to support the regulation of emotions, we need to be able to evaluate the results of this regulation, that is, changes in emotional responding. Researchers generally view emotions as a concurrent change in experiential, behavioural, and physiological systems that organize human motivational behaviour (e.g., Frijda, 1986; Mauss et al., 2005). Thus, our first task is to identify measures that capture a wide view of emotional processes. There have been two research traditions of emotions. The first tradition views emotions as discrete states, such as, disgust, fear, joy, sadness, and surprise (Ekman, 1993). The second tradition views emotions as a three-dimensional space varying in emotional valence, arousal, and dominance (Bradley \& Lang, 1994; 2000). These traditions have direct consequences especially for measuring the experiential component of emotions. For example, one common method is to ask people to rate their experiences using bipolar scales of emotional valence (i.e., from negative to positive), arousal (i.e., from calm to aroused), and dominance (i.e., from feeling of being in control to being controlled).

The measurement of the experiential component of emotion often requires that the person is interrupted and asked to report her or his experiences. For example, during exposure therapy patients are periodically asked to rate the intensity of their anxiety using a scale of subjective units of discomfort (SUD) ranging from 0 to either 10 or 100 (see, e.g., review by Krijn et al., 2004). The rating is used to evaluate when the level of anxiety has changed and requires the therapist to adapt the exposure. When the anxiety is very high, the exposure may be decreased, for example, with instructed relaxation. Low anxiety suggests that the patient is ready to proceed to a higher level of exposure, for example, to take one step closer to a spider. This way, the person is gradually exposed to the object of their fear and habituated to ever increasing amounts of exposure in the process.

The drawback of reporting subjective experiences is that it distracts the person's attention from any ongoing tasks that she or he may be performing. This may hinder a person's experience of being present in the virtual therapy environment. There is some evidence pro the view that this feeling is critical for the success of exposure therapy, as it is required for the experience of relevant emotions and learning to regulate them (Krijn et al., 2004). In this sense, behavioural and physiological components of emotion are somewhat more convenient to measure. It is feasible to acquire these measures continuously and in real-time without distracting the person (Öhman et al., 2000; Teller, 2004; Wilhelm et al., 2006; Mandryk \& Atkins, 2007). This also creates potential for more accurate timing of emotional responses. For example, the exact time of a reaction to some surprising event is more readily identified from changes in facial behaviour as compared to a post study questionnaire. 
Measures of facial behaviour have been frequently used for detecting emotional responses. For example, Ioannou and others (2005) reported results from using an adaptive system to classify the facial behaviour of one person. The system classified emotional facial expressions into three classes based on features extracted from video images. The classes represented three out of four quadrants of a two-dimensional emotional space (i.e., high arousal - negative, high arousal - positive, low arousal - negative). The classification accuracy of a general (i.e., person-independent) model was about $58 \%$. After adapting this model to the particular person, the performance increased to approximately $78 \%$.

In contrast to Ioannou et al. (2005), typically the classes in video-based classification of facial behaviour have been based on a view of discrete emotions (see, e.g., reviews in Donato et al., 1999; Cowie et al., 2001). The accuracies for these kinds of classifiers are impressive at their best. For example, Sohail \& Bhattacharya (2007) reported an average accuracy of over $90 \%$ in classifying six emotional facial expressions. However, discrete classifiers usually do not address the intensity of emotional states which is used in adapting the amount of virtual exposure. As an exception, Bailenson (in press) recently developed a classifier for both the discrete facial expression and the intensity of the expression. In any case, most previously investigated discrete classifiers are limited in their applicability to virtual therapy.

Video-based measures can be used to detect facial activity in a non-invasive manner, for example, without restricting the movements of the person by electrode wires. However, video-based methods can only be used to detect clearly visible facial behaviour, while electrophysiological measures have the potential to register very small changes in muscle activity (Ekman et al., 2002). There is also evidence that physiological measures can reflect emotional responses that do not evoke observable behaviour (e.g., Gross \& Levenson, 1997). Furthermore, video-based measures are very sensitive to lighting and head orientation as well as to inaccuracies in detecting facial landmarks (e.g., Cowie et al., 2005). For these reasons, physiological measures may be seen to reflect a more objective (e.g., contextindependent) view of the emotional response.

A common method for measuring the physiological activity that underlies visible facial behaviour is electromyography (EMG). Facial EMG is performed by attaching electrodes that register the electrical activity of facial muscles over specific muscle sites (Tassinary \& Cacioppo, 2000). Especially the EMG activity of the corrugator supercilii (activated when frowning) and the zygomaticus major (activated when smiling) muscles has been frequently found to co-vary with subjective experiences of emotional valence (e.g., Lang et al., 1993; Larsen et al., 2003). The corrugator supercilii muscle which knits and lowers the brow is located in the forehead. Its activity has been found to increase when a person experiences negative emotions and to decrease during positive experiences. The zygomaticus major is a relatively large muscle located in the cheek. When activated it pulls up the corner of the mouth. The intensity of its activity varies with emotional valence in the opposite manner to the corrugator supercilii muscle.

Although some physiological reactions are quite straight-forward to interpret, humans do not normally evaluate emotional expressions of other people from electrophysiological data. Even one electrophysiological signal can contain lots of information, which may overwhelm a human observer. For example, facial EMG activity may reflect both the intensity of facial muscle activations and the fatigue in muscles (Tassinary \& Cacioppo, 2000).

Automatic analysis and interpretation of physiological signals can help in perceiving which changes in signals are related to emotional processes. There is evidence that even the 
subjective component of an emotion (i.e., emotional experiences) can be automatically estimated from electrical facial muscle activity. For example, Partala and others $(2005 ; 2006)$ were able to build systems that automatically estimated and classified emotional experiences evoked by picture and video stimuli. The first system (Partala et al., 2006) was adapted to the individual responses of each person as follows. First, participants were shown a calibration block of 24 pictures selected from the standardized set of International Affective Picture System (IAPS). After each stimulus, the participant rated the emotional valence that she or he experienced using a 9-point bipolar scale. Then, the statistical models that estimated the emotional valence were adapted to the person based on the ratings and the EMG data from the calibration block. Finally, the system was tested using 28 pictures and six videos that showed a female or a male actor crying, smiling, and portraying a neutral facial expression. Subjective ratings of emotional valence were collected after each stimulus. These ratings and the system's estimate of emotional valence were compared in order to determine the accuracy of the system. The results showed that the best models were able to separate negative and positive emotional responses with accuracies of over 70 percent for pictures and over 80 percent in the case of video stimuli. Further, the largest correlation between the subjective ratings and the system's estimate of emotional valence on a 9-point scale was over 0.9. Thus, the results of the first system showed that subjective emotional experiences can be estimated based on measures of electrical facial activity with relatively simple models in real-time. Although there is still room for improvement, the accuracy achieved in this study is already sufficient for many applications.

The second system was person-independent and therefore did not require a separate calibration period (Partala et al., 2005). The valence of emotional experiences was estimated based on the direction of change in EMG activity from a baseline period of 0.5 seconds before stimulus onset. This system was able to distinguish between reactions rated as positive or negative at an accuracy of nearly 70 percent for pictures and over 80 percent for videos. In summary, facial activity shows clear promise as a reliable measure for automatic, real-time classification of emotional valence, as both person-adapted and personindependent systems were demonstrated to perform at a reasonable accuracy.

In addition to measures of electrical facial muscle activity, there is a wide variety of other physiological measures that have been shown to vary between emotional reactions, such as the mean heart rate and its frequency components (Anttonen \& Surakka, 2005; Levenson \& Ekman, 2002; Bradley, 2000; Malliani et al., 1991). For example, Rainville and others (2006) investigated classification of emotional responses using a large set of heart activity and respiration related features. Participants recalled and experientially relived one or two autobiographical episodes associated with the experience of fear, anger, sadness, or happiness. The system was able to detect which of the four emotions the participant was experiencing (i.e., according to subjective ratings) at an accuracy of about $65 \%$.

One challenge that has rarely been investigated in previous classification studies is the recognition and accurate timing of emotional responses. In other words, participants themselves have typically reported the onset and offset of emotional responses and data has been segmented by hand. It is clear that in order to react to the events in real-time, a system should be able to segment the collected data without human intervention. Vanhala \& Surakka (2007b) recently reported a study of this kind of an online system. The system automatically detected the onset and offset of emotion related events (i.e., voluntary smiling and frowning) based on less than half a second of heart rate data. The onset of activity was 
detected with a statistically significant accuracy of $66.4 \%$ and the offsets were detected with an accuracy of $70.2 \%$. However, the rate of false recognitions was $59.7 \%$ which is quite high. Thus, the results showed that the heart rate can be used to support recognition and classification of emotional responses, but it should be used as one of several corroborative measures in practical applications.

In fact, previous studies have usually employed more than one measure in classifying emotional states (e.g., Kim et al., 2004; D’Mello et al., 2007; Mandryk \& Atkins, 2007). Otherwise, recognizing mental states and responses can be challenging, as physiological responses are person-dependent and they reflect several overlapping reactions and mental processes. For example, Bailenson and others (in press) compared classifiers that used facial activity as such or combined it with several physiological measures of heart activity, skin conductance, and finger temperature. The use of physiological measures significantly improved the precision of classification (i.e., the proportion of correctly classified samples in each classified group) as compared to classifiers that used only hand-coded facial features. The best improvements were over $15 \%$ for classifying sadness and about $9 \%$ for classifying amusement. Similarly, Zeng and others (2004) were able to improve the accuracy of their emotion classification system to 90 percent when both facial expressions and prosodic cues of speech were used. When only one of these modalities was used, the accuracies dropped to 56 and 45 percent, respectively. Busso and others (2004) achieved similar results with a system that recognized emotions from speech and facial expressions. In an earlier work, Picard and others (2001) identified specific physiological responses from four physiological signals (i.e., facial electromyogram, blood volume pressure, skin conductance, and respiration) and used these response patterns in classifying emotional experiences to eight classes. They achieved a classification accuracy of 81 percent.

The measurement of bioelectric signals can be criticized based on the complex arrangements (e.g., electrodes, amplifiers, and skin cleaning) that are required for measuring them. Recently, several wireless and non-invasive technologies have been developed for measuring physiological signals, including facial EMG (e.g., Anttonen \& Surakka, 2005; Teller, 2004; Wilhelm et al., 2006). For example, the electrical activity of forehead muscles (e.g., corrugator supercilii) can be measured with an easy-to-wear wireless headband that contains embroidered silver thread electrodes (Vehkaoja \& Lekkala, 2004; Nöjd et al., 2005). As another example of non-invasive and easily applied measurement technology, Anttonen and Surakka $(2005 ; 2007)$ were able to reliably measure emotion related heart rate changes with a regular looking office chair. The chair contained embedded electromechanical sensors in the seat, arm rests, and back rest. The sensors can be used to detect pressure changes due to heart activity, body movement, or changes in posture. Based on these recent advances in non-invasive technologies, physiological measures are quickly catching up on the current benefits of video-based methods for tracking changes in emotion related behaviour.

In summary, there are several well-tried methods for measuring the different aspects of emotion. Our present review suggested that especially physiological measures show potential as objective and sensitive measures of emotion related processes. Thus, there is no need to rely on any single measure of emotional processes, such as SUD in adjusting the exposure in virtual therapy. In fact, typically several measures have been fused together in order to derive more accurate compound measures. This also helps in interpreting the data, as it can be pre-processed into a form that is more accessible to a human observer. Further, physiological measures are less prone to distract the person as they can be continuously 
acquired without intervention. However, monitoring emotion related processes can still require considerable human effort after integration and interpretation by the computer. The model that we present in the current paper is aimed to facilitate this work.

\section{Regulating emotions with computers}

Social and emotional cues from computers have been found to evoke significant responses in their human observers. For example, synthesized speech with emotional content has been found both to evoke positive emotions and to enhance problem solving activity (Partala \& Surakka, 2004; Aula \& Surakka, 2002). Aula \& Surakka (2002) used synthesized speech to provide neutral, negatively, or positively worded auditory feedback that seemed to reflect participant's performance in solving arithmetic problems. In reality, the content of feedback was random and independent of the participant's performance. Nonetheless, positive feedback significantly facilitated the speed of solving problems. In a later study, Partala \& Surakka (2004) investigated emotionally worded interventions after a pre-programmed mouse delay during computerized puzzle solving tasks. Similar to the previous study, problem solving performance was significantly better after positively worded interventions. In terms of facial EMG measurements, participants also smiled more and frowned less after positive interventions as compared to facial activity after neutral and negative interventions. These kinds of studies have shown that explicit feedback and interventions from computers can affect human cognitive and emotional processes. There is also evidence that even more subtle social and emotional cues are effective in human-computer interaction. For example, in one of the first studies of virtual proximity, Partala and others (2004) investigated reactions to the simulated distance of a virtual head. When the head appeared to be closer, participants rated that they felt dominated by it. Vice versa, when the head was further away, participants felt that they were controlling it. Vanhala and others (submitted) recently found similar subjective dominance reactions to the simulated proximity of an embodied computer agent. Some researchers have even described computers as social actors, meaning that people have a strong tendency to behave socially when interacting with computers (Nass et al., 1994; Reeves \& Nass, 1996).

The effectiveness of virtual stimuli in evoking social and emotional reactions is the basis for virtual exposure therapy. The idea is that new neutral memory structures are formed during virtual exposure. These memory structures should replace the previous anxiety related structures when responding to real-life situations (Krijn et al., 2004). In other words, people should react to provoking virtual stimuli in the same manner as to authentic, real-life stimuli. There are some studies that support his view. For example, socially anxious people get highly distressed when they talk to or need to disturb embodied artificial characters in virtual reality (Pertaub et al., 2002; Garau et al., 2005). Further, the effects of virtual exposure to spiders have been found to generalize to real-life behaviour as measured by the Behavioural Avoidance Test (Garcia-Palacios et al., 2002). That is, people were able to approach a real spider more easily after exposure to a virtual one.

In addition to these computer generated stimuli that regulate emotional responses, emotions can also be actively self-regulated. Gross \& Thompson (2007) have described the development of emotion self-regulation as a continuum. In the first stages emotions are consciously regulated. Later, emotion regulation becomes more automatic and effortless. Thus, the process of learning to regulate emotions resembles the process of skill acquisition in general (Anderson, 2000). In this view, less skilled emotion regulators use cognitive 
processes extensively to support the regulation. For example, they may deliberately rely on instructions and examples of successful regulation. After practise, the regulation of emotions becomes autonomous and efficient, demanding much less cognitive processing. For example, a skilled self-regulator does not need to explicitly apply instructions (e.g., from a therapist) in order to regulate emotions.

Instructions that support emotion regulation may be relatively simple. For example, Vanhala \& Surakka (2007a) investigated whether computer-guided voluntary facial activations have an effect on autonomous nervous system activity. Participants were instructed to activate either the corrugator supercilii muscle or the zygomaticus major muscle at one of three intensity levels (i.e., low, medium, or high). Instructions for each task and realtime feedback about the intensity of facial muscle activations were provided to the participant on the computer screen. Subjective ratings of emotional valence were collected after the activation. It was found that different muscle activations produced both taskspecific emotional experiences and significant changes in heart rate and heart rate variability. Thus, the results showed that relatively simple computer-instructions allow people to actively influence their involuntary physiological reactions and subjective experiences that are associated with emotions.

Physiological feedback as such can also help in learning to regulate emotions. Usually, either skin conductivity or breathing patterns are registered and displayed to the patient or the therapist during computer-assisted therapy sessions (Wiederhold \& Bullinger, 2005). This way, a person can become aware of unconscious physiological responses and processes, which can enable voluntary control over them. As an impressive example in favour of the effectiveness of virtual exposure therapy, Wiederhold \& Wiederhold (2003) followed the behaviour of a group of 10 patients inflicted with fear of flying who were treated using virtual exposure and physiological feedback. As the terrorist attacks on September $11^{\text {th }}, 2001$ were quite directly related to flying, they could have caused relapses in terms of intensifying the fear of flying in these patients. However, everyone of this group was able to fly without medication or further treatment just four months after the attacks.

Physiological data can also be collected for later reflection. For example, Lindström and others (2006) presented an "affective diary" that provided a multimodal (i.e., auditory and visual) representation of sensor data. A measure of arousal was extracted from the physiological measures and the estimated level of arousal affected the posture of a virtual character displayed on the screen. Users of the diary could later reflect their experiences and manipulate the character in order to match it to their recollection of those feelings. This application illustrates the interplay of involuntary emotion related physiological reactions (i.e., visually coded sensor data) and voluntary regulation of emotions (i.e., later reflection and adjustment in "affective diary"). However, a crucial component for supporting the training of emotion regulation is the online adjustment of emotional stimulation, for example, the amount of exposure to virtual stimuli. This requires a real-time system for the evaluation and reflection of psychological and physiological processes.

In summary, computer systems show potential for regulating human emotions. First, studies have shown that people react socially and emotionally to computers and virtual environments. Second, the effects of virtual stimuli (e.g., habituation of anxiety responses) have been further facilitated when feedback of emotion related physiological activity has been provided. Third, we found that voluntary regulation of emotion related processes seems to be a potential key factor both in learning the regulation as such but also in 
modulating the functioning of involuntary mechanisms activated during emotion related processes. Fourth, we reviewed a large number of physiological measures that show potential as sensitive and objective measures of emotional responses. The relevant information from these measures could be extracted using automatic classification of emotional responses. This way, it would be possible to avoid overwhelming a human observer, while still using all of the available information in order to maximally support emotion regulation. In the next section we present a model that supports this goal by integrating perceptual intelligence to the system.

\section{Adaptive support for emotion regulation}

Figure 1 shows a model of how virtual exposure therapy is currently performed. The model contains a set of different actors that take part in an interaction loop. First, the relevant emotional state is observed using different emotion related measures. Then, a human facilitator monitoring these measures decides how the virtual stimuli should be adapted. For example, if the patient reports a relatively low subjective experience of discomfort, the facilitator may proceed to increase the amount of exposure, for example, by moving a virtual spider closer. Note that the facilitator may in fact be the person who is being measured and treated, that is, the person may choose to control the amount of stimulation her or himself. Finally, the interaction loop in Figure 1 is closed after the adaptation by the newly modified stimulation. For example, if the virtual spider was moved closer, it may now provoke stronger anxiety reactions. These anxiety reactions are then reflected in the measures of emotion related processes, which leads to another cycle of interaction. The underlying idea of these continuous cycles is that the person learns to regulate emotional responses to increasing levels of stimulation.

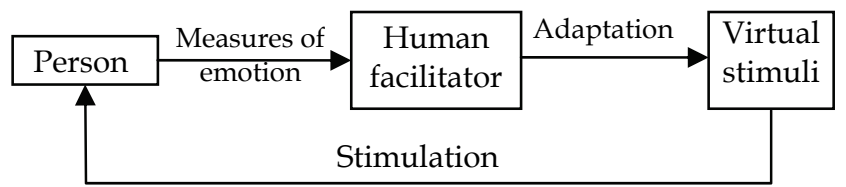

Fig. 1. A diagram of the current model used in virtual exposure therapy. Different actors are presented as boxes and labelled arrows represent the flow of information.

Although the model is quite compact and straight-forward, there are three major challenges involved when it is applied. First, although previous work has shown that virtual stimulation is effective in evoking similar emotional and social responses as real-life stimuli, the effects of virtual stimulation and its online adaptation have not been extensively investigated. It has also been found that computer-generated stimuli may significantly facilitate cognitive processing and effectively support regulation of anxiety responses. However, more information is still needed about how adapting the different parameters of stimulation in real-time affects emotion related processes. This challenge should be resolved by controlled experimental studies of each virtual stimulus in the future.

The second challenge is that there are several emotion related measures that provide complementary, non-overlapping information. There is a large amount of information 
contained in each of these measures. A human observer is often forced to choose between a broad view of the emotional state and an in depth analysis of it. Perceptual intelligence can be used to solve this challenge. Different methods for building computers that perceive emotion from physiological and behavioural measures were reviewed in the second section. These methods form the basis for the perceptual intelligence in our new model.

Figure 2 presents a model where perceptual intelligence has been included into the system. The model is similar to the previous one with the exception that the interpretation of emotion related measures is performed automatically. Thus, the facilitator has access to a higher level representation or a summary of information that is relevant to therapy. Simply stated, the computer acts as a kind of a translator that deciphers the information in the measured signals into a summary that is more accessible to the human observer. This way, the facilitator is less likely to be overwhelmed with the load of information available from different experiential, behavioural, and physiological measures. However, the actual adaptation is still controlled by a human facilitator acting on the basis of the summarized information.

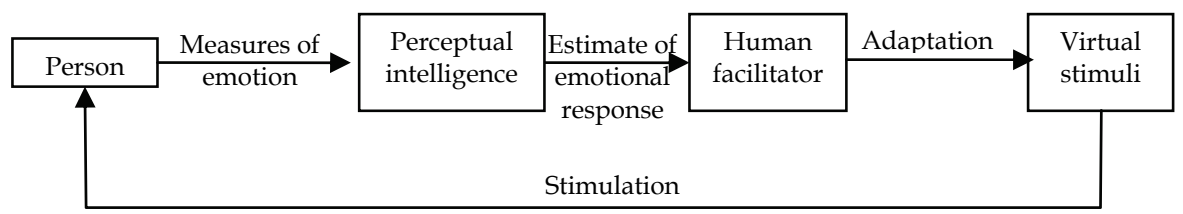

Fig. 2. A diagram of a perceptually intelligent model to be used in virtual exposure therapy.

Different actors are presented as boxes and labelled arrows represent the flow of information.

The third and final challenge in using the conventional model is that it places the human facilitator as a part of the real-time system (see Figs. 1\&2). This requires that a person must continuously attend to the measurements and decide when and how to react to any changes or even to a lack of changes. Figure 3 shows a final model designed to more efficiently support emotion regulation in virtual exposure therapy. The continuous monitoring of emotion related information is now built into the computer system itself. In contrast to conventional computer systems that place humans as a part of the processing loop, this model can support emotion regulation without distracting the person or requiring constant attention. The system provides this support by taking the initiative and adapting the stimulation when it is appropriate, that is, by being proactive (Tennenhouse, 2000).

In this kind of a system, the role of the human facilitator is to supervise the process of therapy. In order to perform this task, the supervisor needs information about the therapy process and the functioning of the system. Further, this information should be concise if we are to retain the main advantage of automatic signal analysis and reasoning which was to allow people to focus on the task at hand. One potentially efficient way to do this is to provide an explanation of the system's reasoning to the supervisor. This type of a model fits the definition of an expert system which solves problems in a narrow domain of expertise (i.e., virtual exposure therapy) and is able to explain its own reasoning (Bratko, 2001). For example, if the system moved the object of the phobia closer to the person, it could be asked why it did this. A brief explanation could be that, for instance, the physiological signals showed that the current level of anxiety was very low. Then, the person could further query 
the specifics of these signals, if she or he so desires. This way, the users confidence of the system's functioning could be increased by making it transparent to the user. Of course, this would also allow the system's reasoning to be monitored and tuned when appropriate.

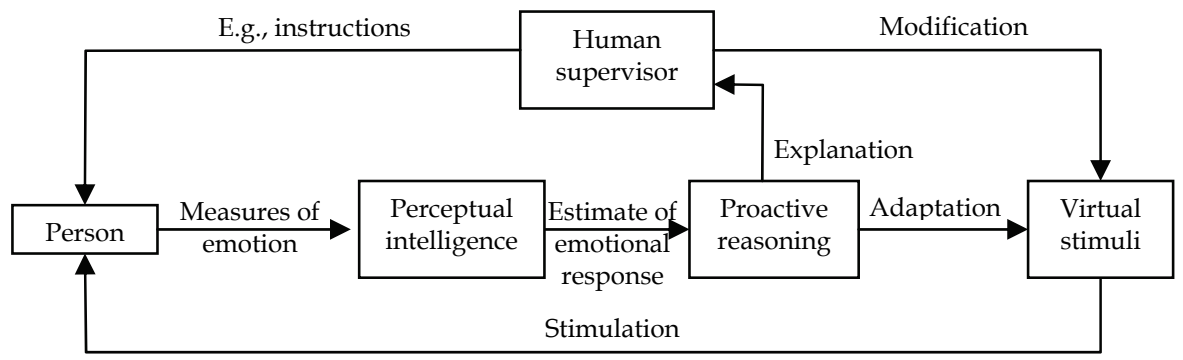

Fig. 3. A diagram of a proactive model for virtual exposure therapy. Different actors are presented as boxes and labelled arrows represent the flow of information.

During the operation of the system its supervisor may exert control over the system either by adapting the stimulation directly or through interaction with the person who is being trained. For example, a therapist may instruct the person to relax by performing controlled breathing exercises. This voluntary control affects the physiological state which is further reflected in the collected emotion related measures. As a result, the changes in these measures lead to corresponding adaptation of the stimulation. As another example, the person may directly influence those measures that affect the intensity of stimulation. This is feasible as the same expressive channels that reflect spontaneous emotional reactions can also be voluntarily controlled. For instance, the person may voluntarily frown in order to signal a high level of discomfort and move the spider further away. This also highlights another advantage of proactive adaptation. As responses to measured changes (i.e., voluntary activity) are explicit pre-programmed reactions, they can be guaranteed to be consistent. This might not be the case if the responses were selected by a human operator. In summary, a perceptually intelligent and proactive system enables a wide variety of information to be used in regulating emotions. First, perceptual intelligence enables more efficient processing of measured emotion related signals. This enables the monitoring of a larger set of emotional measures, which then results in a more comprehensive and reliable view of the emotional state, for example, attending to multiple physiological and behavioural changes. Second, proactive reasoning may be used to adapt the stimulation in an appropriate and consistent manner. The adaptation can be based on findings that show how virtual stimulation affects human emotions and cognitive processing. As a whole, a system that uses this model can function independently without constant human supervision, helping people to regulate emotions without distracting them.

\section{Discussion and future work}

The current work presented a model for a computer system that supports the regulation of emotion related processes during exposure to provoking stimuli. We identified two main challenges for these kinds of systems. First, emotions as such are complex, multi-component processes that are measured with several complementary methods. The amount of 
information can overwhelm a human operator. Second, the adaptation of stimulation requires real-time reasoning about the current emotional state and the effects of adaptation. This reasoning may distract a human facilitator from tasks related to emotion regulation. Further, a human operator may respond inconsistently to changes in emotional processes, which effectively removes the control of the system from the person who is being trained.

In the present work we addressed the first challenge of identifying emotional reactions by including perceptual intelligence to our model. Several measures for automatic analysis of emotional state have been investigated in previous studies. Especially physiological measures were found to show potential as objective and reliable measures of emotional processes. For example, there are several new wireless and wearable measurement technologies that enable continuous and non-invasive measurement of emotion related physiology. Thus, automatic analysis of emotion related physiological activity can help to identify significant emotional responses during virtual exposure therapy. For a human observer, this pre-processed data is easier to interpret and apply to emotion regulation.

The second challenge of adapting virtual stimulation was addressed with proactive reasoning. First, we reviewed studies of human responses to virtual stimulation. These studies showed that human cognitive functioning and emotional responses may be significantly regulated using different computer-generated social and emotional cues, for example, virtual proximity. Second, we suggested a model where the computer automatically adapts the virtual stimulation according to the emotional state that it has perceived. This way, perceptual intelligence and artificial reasoning result in a proactive system that does not require humans to process data in real-time. In other words, when our model is applied to virtual exposure therapy, a person can focus on the training itself instead of monitoring and responding to measured physiological signals.

In spite of the promising findings from previous studies, there are still open questions in the computer perception of emotional responses to provoking stimuli. For example, some findings suggest that physiological reactions of phobics and healthy people may be significantly different (Wilhelm \& Roth, 1998). Although the responses may be similar in terms of direction of change from a baseline (e.g., heart rate accelerated in both phobics and healthy subjects exposed to provoking stimulation), the differences in the magnitude of change may affect the results of automatic recognition. This raises the question whether automatic classification methods for emotional responses in healthy people provide information that is applicable to treating emotional disorders (i.e., abnormal emotional responses). Thus, there is a need to study systems where automatic perception has been included in a virtual therapy system.

The previous research on automatic classification of emotional states has used both personindependent methods and methods that are calibrated to each individual person. These two types of methods are suited for different kinds of applications (Bailenson et al., in press). Systems based on a universal model of emotional responses are suited when lots of people use the same interface, for example, a public computer at a library. An idiosyncratic model that adapts to each person is more suitable when the same person repeatedly uses the interface. The latter case is typical in virtual therapy applications, as the person is treated over multiple similar sessions (Krijn et al., 2004; Wiederhold \& Wiederhold, 2005). However, a person-independent model could be used as a starting point for the adaptation, similar to the video-based system by Ioannou and others (2005). This would enable the system to provide estimates of emotional experiences even before a set of person-specific physiological 
data is collected and calibration is performed. Then, later adaptation of the model could be performed to improve its accuracy.

Another challenge in perceptual intelligence that has received little attention is the automatic segmentation of collected measures. Most previous studies of automatic classification of emotional responses have used hand-segmented data. Typically, a participant reports the onset and the offset of an emotional state and the data is segmented off-line. In contrast to these methods, virtual exposure therapy requires a system that analyses the data online and adapts to the emotional state of a person in real-time. If perceptual intelligence is to be included in this kind of a system, there is a need to investigate online, automatic segmentation of physiological data. Our preliminary results of heart rate responses have shown that such automatic segmentation is feasible (Vanhala \& Surakka, 2007b). However, there is a need to investigate systems that use multiple complementary signals in order to improve the reliability and accuracy of methods.

On a general level, our review suggested that people appreciate computer systems that respond to their emotions, for example, display empathy (Klein et al., 2002; Brave et al., 2005). Although it seems a small step to assume that people would appreciate computers that administer virtual exposure therapy by responding to anxiety, there can be a fundamental difference. Emotion regulation aims not only to respond but also to change the emotional reactions to virtual and real-life emotional stimulation. There is a need to study how people experience this kind of a system and whether it helps in regulating emotions.

In summary, the present work showed how automatic perception of emotions and proactive adaptation of a computer system could help in facilitating virtual exposure therapy. The skill of regulating emotions is gradually acquired by adapting virtual stimuli according to the emotional state of the person. This principle is applicable to other emotionally intelligent applications as well. For example, we might be less likely to loose our temper if the desktop computer could display empathy when an important document gets accidentally deleted. Thus, research on perceptual intelligence and proactive reasoning in virtual exposure therapy systems has the potential to improve the quality of human-technology interaction in general. The current work identified the state-of-the-art and the future research that will help in reaching this goal.

\section{Acknowledgements}

This research was financially supported by the Graduate School in User-Centered Information Technology and the Academy of Finland (project number 1115997).

\section{References}

Anderson, J. R. (1999). Learning and memory: An integrated approach, Wiley, New York.

Anttonen, J. \& Surakka, V. (2005). Emotions and heart rate while sitting on a chair, Proceedings of CHI 2005, 491-499, ACM.

Anttonen, J. \& Surakka, V. (2007). Music, heart rate, and emotions in the context of stimulating technologies, Affective Computing and Intelligent Interaction, LNCS, 4738, 290-301.

Anxiety Disorders Association of America. (2008). Statistics and facts about anxiety disorders, http://www.adaa.org/ AboutADAA/PressRoom/Stats\&Facts.asp, Jan 30th, 2008.

Aula, A. \& Surakka, V. (2002). Auditory Emotional Feedback Facilitates Human-Computer Interaction, Proceedings of HCI 2002, 337-349, Springer. 
Bailenson, J. N.; Pontikakis, E. D.; Mauss, I. B.; Gross, J. J.; Jabon, M. E.; Hutcherson, C. A.; Nass, C. \& John, O. (in press). Real-time classification of evoked emotions using facial feature tracking and physiological responses. International Journal of HumanComputer Studies.

Bradley, M. M. (2000). Emotion and Motivation, In: Handbook of Psychophysiology, Cacioppo, J. T.; Tassinary, L. G. \& Berntson, G. G. (Ed.), 602-642, Cambridge University Press.

Bradley, M. M. \& Lang, P. J. (2000). Affective reactions to acoustic stimuli. Psychophysiology, 37, 204-215.

Bradley, M. M. \& Lang, P. J. (1994). Measuring emotions: the self-assessment manikin and the semantic differential. Journal of Behavioral Therapy and Experimental Psychiatry, $25,1,49-59$.

Bratko, I. (2001). Prolog Programming for Artificial Intelligence, 3rd ed., Pearson.

Brave, S.; Nass, C. \& Hutchinson, K. (2005). Computers that care: investigating the effects of orientation of emotion exhibited by an embodied computer agent. International Journal of Human-Computer Studies, 62, 161-178.

Busso, C.; Deng, Z.; Yildirim, S.; Bulut, M.; Lee, C. M.; Kazemzadeh, A.; Lee, S.; Neumann, U. \& Narayanan, S. (2004). Analysis of emotion recognition using facial expressions, speech and multimodal information, Proceedings of ICMI, 205-211.

Cowie, R.; Douglas-Cowie, E.; Taylor, J.; Ioannou, S.; Wallace, M. \& Kollias, S. (2005). An intelligent system for facial emotion recognition, Proceedings of IEEE ICME, $4 \mathrm{pp}$.

Cowie, R.; Douglas-Cowie, E.; Tsapatsoulis, N.; Votsis, G.; Kollias, S.; Fellenz, W. \& Taylor, J. (2001). Emotion Recognition in Human-Computer Interaction. IEEE Signal Processing Magazine, 18, 1, 32-80.

D'Mello, S.; Graesser, A. \& Picard, R. W. (2007). Toward an affect-sensitive AutoTutor. IEEE Intelligent Systems, 22, 4, 53-61.

Donato, G.; Bartlett, M. S.; Hager, J. C.; Ekman, P. \& Sejnowski, T. J. (1999). Classifying facial actions. IEEE Transactions on Pattern Analysis and Machine Intelligence, 21, 974-989.

Ekman, P. (1993). An argument for basic emotions. Cognition and Emotion, 6, 3, 169-200.

Ekman, P.; Friesen, W. V. \& Hager, J. C. (2002). Facial Action Coding System: Investigator's Guide, A Human Face, Salt Lake City, USA.

Frijda, N. H. (1986). The Emotions, Cambridge University Press.

Garau, M.; Slater, M.; Pertaub, D. \& Razzaque, S. (2005). The Responses of People to Virtual Humans in an Immersive Virtual Environment. Presence: Teleoperators E Virtual Environments, 14, 1, 104-116.

Garcia-Palacios, A.; Hoffman, H.; Carlin, A.; Furness, T. A. \& Botella, C. (2002). Virtual reality in the treatment of spider phobia: a controlled study. Behaviour Research and Therapy, 40, 9, 983-993.

Gross, J. J. \& Levenson, R. W. (1997). Hiding feelings: The acute effects of inhibiting negative and positive emotion. Journal of Abnormal Psychology, 106, 1, 95-103.

Gross, J. J. \& Thompson, R. A. (2007). Emotion regulation: Conceptual foundations, In: Handbook of Emotion Regulation, Gross, J. J. (Ed.), 3-24, Guilford Press.

Ioannou, S. V.; Raouzaiou, A. T.; Tzouvaras, V. A.; Mailis, T. P.; Karpouzis, K. C. \& Kollias, S. D. (2005). Emotion recognition through facial expression analysis based on a neurofuzzy network. Neural Networks, 18, 4, 423-435. 
Kim, K. H.; Bang, S. W. \& Kim, S. R. (2004). Emotion recognition system using short-term monitoring of physiological signals. Medical and Biomedical Engineering and Computing, 42, 3, 419-427.

Klein, J.; Moon, Y. \& Picard, R. W. (2002). This computer responds to user frustration: Theory, design, and results. Interacting with Computers, 14, 119-140.

Krijn, M.; Emmelkamp, P.; Olafsson, R. \& Biemond, R. (2004). Virtual reality exposure therapy of anxiety disorders: A review. Clinical Psychology Review, 24, 259-281.

Lang, P. J.; Greenwald, M. K.; Bradley, M. M. \& Hamm, A. O. (1993). Looking at pictures: Affective, facial, visceral, and behavioral reactions. Psychophysiology, 30, 261-273.

Larsen, J. T.; Norris, C. J. \& Cacioppo, J. T. (2003). Effects of positive and negative affect on electromyographic activity over the zygomaticus major and corrugator supercilii. Psychophysiology, 40, 776-785.

Levenson, R. W. \& Ekman, P. (2002). Difficulty does not account for emotion-specific heart rate changes in the directed facial action task. Psychophysiology, 39, 397-405.

Lindström, M.; St, A.; Höök, K.; Sundström, P.; Laaksolahti, J.; Combetto, M.; Taylor, A. \& Bresin, R. (2006). Affective diary: designing for bodily expressiveness and selfreflection, CHI '06 extended abstracts, 1037-1042, ACM, New York.

Malliani, A.; Pagani, M.; Lombardi, F. \& Cerutti, S. (1991). Cardiovascular neural regulation explored in the frequency domain. Circulation, 84, 482-492.

Mandryk, R. L. \& Atkins, M. S. (2007). A fuzzy physiological approach for continuously modeling emotion during interaction with play technologies. International Journal of Human-Computer Studies, 66, 329-347.

Mauss, I. B.; Levenson, R. W.; McCarter, L.; Wilhelm, F. H. \& Gross, J. J. (2005). The tie that binds? Coherence among emotion experience, behavior, and physiology. Emotion, 5, 2, 175-190.

Nass, S.; Steuer, J. \& Tauber, E. R. (1994). Computers are Social Actors, Proceedings of CHI'94, 72-78.

Nöjd, N.; Puurtinen, M.; Niemenlehto, P.; Vehkaoja, A.; Verho, J.; Vanhala, T.; Hyttinen, J.; Juhola, M.; Lekkala, J. \& Surakka, V. (2005). Wireless wearable EMG and EOG measurement system for psychophysiological applications, Proceedings of the 13th Nordic Baltic Conference on Biomedical Engineering and Medical Physics, 144-145

Öhman, A.; Hamm, A. \& Hugdahl, K. (2000), Cognition and the Autonomic Nervous System: Orienting, Anticipation, and Conditioning, In: Handbook of Psychophysiology, Cacioppo, J. T.; Tassinary, L. G. \& Berntson, G. G. (Ed.), 533-575.

Partala, T. \& Surakka, V. (2004). The effects of affective interventions in human-computer interaction. Interacting with Computers, 16, 2, 295-309.

Partala, T.; Surakka, V. \& Vanhala, T. (2006). Real-time estimation of emotional experiences from facial expressions. Interacting with Computers, 18, 208-226.

Partala, T.; Surakka, V. \& Vanhala, T. (2005). Person-independent estimation of emotional experiences from facial expressions, Proceedings of IUI 2005, 246-248, ACM.

Pentland, A. (2000). Perceptual intelligence. Communications of the ACM, 43, 3, 35-44.

Pertaub, D.; Slater, M. \& Barker, C. (2002). An Experiment on Public Speaking Anxiety in Response to Three Different Types of Virtual Audience. Presence: Teleoperators $\mathcal{E}$ Virtual Environments, 11, 1, 68-78. 
Picard, R. W.; Vyzas, E. \& Healey, J. (2001). Toward machine emotional intelligence: Analysis of affective physiological state. IEEE Transactions on Pattern Analysis and Machine Intelligence, 23, 1175-1191.

Rainville, P.; Bechara, A.; Naqvi, N. \& Damasio, A. R. (2006). Basic emotions are associated with distinct patterns of cardiorespiratory activity. International Journal of Psychophysiology, 61, 5-18.

Reeves, B. \& Nass, C. (1996). The Media Equation: How People Treat Computers, Television, and New Media Like Real People and Places, Cambridge University Press, New York.

Sohail, A. S. M. \& Bhattacharya, P. (2007). Classification of Facial Expressions Using KNearest Neighbor Classifier, Proceedings of MIRAGE 2007, 555-566, Springer.

Surakka, V. \& Vanhala, T. (accepted). Emotions in human-computer interaction, In: Multichannel communication on the Internet, Kappas, A. (ed.), Cambridge University Press.

Surakka, V.; Tenhunen-Eskelinen, M.; Hietanen, J. K. \& Sams, M. (1998). Modulation of human auditory information processing by visual emotional stimuli. Cognitive Brain Research, 7, 159-163.

Tassinary, L. G. \& Cacioppo, J. T. (2000). The skeletomotor system: Surface electromyography, In: The Handbook of Psychophysiology, Cacioppo, J. T.; Tassinary, L. G. \& Berntson, G. G. (Ed.), 163-199, Cambridge University Press.

Teller, A. (2004). A platform for wearable physiological computing. Interacting with Computers, 16, 917-937.

Tennenhouse, D. (2000). Proactive computing. Communications of the ACM, 43, 5, 43-50.

Vanhala, T. \& Surakka, V. (submitted). Virtual proximity and facial expressions regulate human emotions and attention. International Journal of Human-Computer Studies.

Vanhala, T. \& Surakka, V. (2007a). Facial activation control effect (FACE), Affective Computing and Intelligent Interaction, Lecture Notes in Computer Science, 4738, 278-289.

Vanhala, T. \& Surakka, V. (2007b). Recognizing the Effects of Voluntary Facial Activations Using Heart Rate Patterns, Proc. of the 11th WSEAS Int. Conf. on Computers, 628-632.

Vehkaoja, A. \& Lekkala, J. (2004). Wearable wireless biopotential measurement device, Proceedings of the IEEE EMBS 2004, 2177-2179.

Ward, R. D. \& Marsden, P. H. (2003). Physiological responses to different WEB page designs. International Journal of Human-Computer Studies, 59, 199-212.

Weiten, W. (2007). Psychology: Themes and variations, Thomson.

Wiederhold, B. K. \& Bullinger, A. H. (2005). Virtual reality exposure for phobias, panic disorder, and posttraumatic disorder: A brief sampling of the literature, Proceedings of HCII 2005, CD-ROM, Lawrence Erlbaum Associates.

Wiederhold, B. K. \& Wiederhold, M. D. (2003). Three-Year Follow-Up for Virtual Reality Exposure for Fear of Flying. CyberPsychology and Behavior, 6, 441-445.

Wilhelm, F. H.; Pfaltzl, M. C. \& Grossman, P. (2006). Continuous electronic data capture of physiology, behavior and experience in real life: towards ecological assessment of emotion. Interacting with Computers, 18, 171-186.

Wilhelm, F. H. \& Roth, W. T. (1998). Taking the laboratory to the skies: Ambulatory assessment of self-report, autonomic, and respiratory responses in flying phobia. Psychophysiology, 35, 5, 596-606.

Zeng, Z.; Tu, J.; Zhang, T.; Rizzolo, N.; Zhang, Z.; Huang, T. S.; Roth, D. \& Levinson, S. (2004). Bimodal HCI-related affect recognition, Proceedings of ICMI, 205-211. 


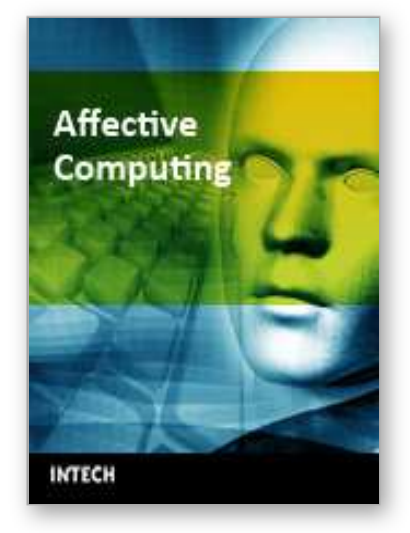

\author{
Affective Computing \\ Edited by Jimmy Or
}

ISBN 978-3-902613-23-3

Hard cover, 284 pages

Publisher I-Tech Education and Publishing

Published online 01, May, 2008

Published in print edition May, 2008

This book provides an overview of state of the art research in Affective Computing. It presents new ideas, original results and practical experiences in this increasingly important research field. The book consists of 23 chapters categorized into four sections. Since one of the most important means of human communication is facial expression, the first section of this book (Chapters 1 to 7) presents a research on synthesis and recognition of facial expressions. Given that we not only use the face but also body movements to express ourselves, in the second section (Chapters 8 to 11 ) we present a research on perception and generation of emotional expressions by using full-body motions. The third section of the book (Chapters 12 to 16) presents computational models on emotion, as well as findings from neuroscience research. In the last section of the book (Chapters 17 to 22) we present applications related to affective computing.

\title{
How to reference
}

In order to correctly reference this scholarly work, feel free to copy and paste the following:

Toni Vanhala and Veikko Surakka (2008). Computer-Assisted Regulation of Emotional and Social Processes, Affective Computing, Jimmy Or (Ed.), ISBN: 978-3-902613-23-3, InTech, Available from: http://www.intechopen.com/books/affective_computing/computerassisted_regulation_of_emotional_and_social_processes

\section{INTECH}

open science | open minds

\author{
InTech Europe \\ University Campus STeP Ri \\ Slavka Krautzeka 83/A \\ 51000 Rijeka, Croatia \\ Phone: +385 (51) 770447 \\ Fax: +385 (51) 686166 \\ www.intechopen.com
}

\author{
InTech China \\ Unit 405, Office Block, Hotel Equatorial Shanghai \\ No.65, Yan An Road (West), Shanghai, 200040, China \\ 中国上海市延安西路65号上海国际贵都大饭店办公楼405单元 \\ Phone: +86-21-62489820 \\ Fax: +86-21-62489821
}


(C) 2008 The Author(s). Licensee IntechOpen. This chapter is distributed under the terms of the Creative Commons Attribution-NonCommercialShareAlike-3.0 License, which permits use, distribution and reproduction for non-commercial purposes, provided the original is properly cited and derivative works building on this content are distributed under the same license. 